\title{
Analysis of Female Breast Shape Based on 3D Human Body Scan in Shanghai
}

\author{
Chen Sheng Nan, Li Yan Mei* \\ Email address: \\ lym0350@126.com (Li Yan Mei) \\ *Corresponding author
}

Fashion Design and Engineering, Shanghai University of Engineering Science, Shanghai, China

\section{To cite this article:}

Chen Sheng Nan, Li Yan Mei. Analysis of Female Breast Shape Based on 3D Human Body Scan in Shanghai. International Journal of Biomedical Science and Engineering. Vol. 4, No. 4, 2016, pp. 34-39. doi: 10.11648/j.ijbse.20160404.11

Received: October 13, 2016; Accepted: October 27, 2016; Published: December 26, 2016

\begin{abstract}
Through three-dimensional human body scanning, randomly selected from Shanghai 223 young women of 3D scanning measurement data, bra sizes standard to classify the samples according to the screened data represent strong 75 women in the chest. Using SPSS mathematical statistical analysis software, the 75 samples were analyzed by cluster analysis, the 75 samples were divided into three categories, and the chest characteristic parameters of each class were summarized. According to the degree of chest width, breast relative height, breast gather degree can be found breast shape of young women in the Shanghai area as a whole, which has a certain degree of ptosis, external expansion, but the degree is not serious can be modified by wearing the right bra of adjustment and to ensure to breast appearance.
\end{abstract}

Keywords: 3D Human Body Scan, Shanghai Women, Chest Shape, Characteristic Parameter

\section{Introduction}

Breast is an important part of female body structure, and it is also an important symbol of female body beauty. With the increase of women's age, in the case of skin relaxation and gland atrophy, the breast gland tissue and adipose tissue is very easy to relax, so as to affect the overall proportion of the breast. Reasonable design of bra structure can give proper support to women's breasts, helps to maintain the beautiful breast shape. Some important factors affecting [1] Xing Yuan mentioned bra wearing fitness and the comfort of the structure and size of the bra, unreasonable structure design or inappropriate size will result in does not fit, so as to cause physical discomfort. The change of Liang Suzhen study by [2] et al. Most of the style and shape of the bra underwear enterprises are based on the basic pattern of bra cup, achieved through tears, darts, transfer and heart-shaped level and width change, so the basic bra pattern design is directly related to body size on the basis of the rationality of bra pattern design.

Shanghai as the world's window, people's living standards are also getting higher and higher, focusing on breast health and breast form the United States are also more and more women. In this paper, we mainly study the breast shape of women in Shanghai area, and measure the size of the main body of the human body, and provide the basis for the reasonable design of the bra structure.

\subsection{Bra Structure}

Bra support, protection, health, fix the body vital role in female somatic play. A female underwear bra is mainly composed of a heart-shaped, and side than in grilled, than, cups, hook and other parts. According to the cup, the ordinary bra is divided into full cup, 3/4 cup, 1/4 cup and half cup; according to the classification of steel ring bra, divided into a steel ring bra and rims bra two [3].

\subsection{Bra Size}

In bra pattern design and specification of the bra plays a vital role in pattern design, product quality inspection is the basis and essential zoom size. Between different countries different standards, China's bra size method is on the chest circumference and cup size as the basis. Reference "People's Republic of China textile industry standards, bra 73012-2008 FZ/T", the bra is said to be: $80 \mathrm{~A}, 85 \mathrm{~B}$, etc.. The bra is digital, is human bust girth size $80 \mathrm{~cm}, 85 \mathrm{~cm}$, human breast milk under the bottom level circumference measurements for a week; the 
bra type is represented by the letter A, B, C, D, E, [4] on human letters represent the difference under the bust. Division of types of bra cup are given in table 1 :

Table 1. Division of bra cup type.

\begin{tabular}{ll}
\hline bra cup types & The difference of chest down \\
\hline AAbra cup & $<10 \mathrm{~cm}$ \\
Abra cup & $10 \mathrm{~cm}$ \\
Bbra cup & $12.5 \mathrm{~cm}$ \\
Cbra cup & $15 \mathrm{~cm}$ \\
Dbra cup & $17.5 \mathrm{~cm}$ \\
Ebra cup & $20 \mathrm{~cm}$ \\
\hline
\end{tabular}

China is currently in the development of bra size shapes, mainly used to determine the bra size under the bust and bust, through the literature and found that the method to determine the bra size does not have scientific and accurate full use of the bust and bust, many try wearing comfortable fit the actual wearing feeling the bra and use the bust and bust difference calculated sizes are not the same, but the same try wearing different styles, structure, fabric bra, will appear the size deviation. So to make a bra more comfortable fit, must take into account the detail size and characteristic parameters of more accurate and detailed for the female breast, chest [5] can accurately reflect the characteristic index and morphological characteristics of the female form of breasts.

\subsection{Problems in the Structure of the Bra}

\subsubsection{Scientific Problem of Plate Making}

In the bra production, Haggar chest plate [6] method is proposed in which overlap to bust bust is equal to $1 / 2$ before and after the prototype prototype before and after the side seam, make circumference than other methods in processing plate side seam, before or after any minus a margin is more scientific and reasonable. But there are still many uncertain problems in the relationship between the structure parameters of the bra and the size of the female body.

The commonly used method of drawing a set number of prototype bra like version [7] is mainly based on the characteristics of bra, the empirical data used to use traditional bra structure design, to be based in the bottom of the cup rim on the form of empirical numerical adjustment.

\subsubsection{Based on the Lack of Cup Volume}

Draw in the use of prototype bra skirts the prototype, the control design of cup capacity, general habits will province near the thoracic thoracolumbar double amplification, and not according to the human body data model, calculate the cup capacity of science, design principle failed to reflect the important parts of prototype bra: parameter by female a prototype of the derived prototype bra for confining the important characteristics of the size of the parts of the bust, chest under control, [8] design experience all of the data are from within the industry.

\subsubsection{Unreasonable Chest High Position}

The bra chest high position is the priority among priorities of cup style design and the design of underwear structure, determines whether to promote good breast bra. But in most of the domestic prototype bra making method are based on the normal position of the coat body as a chest high bra, bra and chest shape function is to adjust clothing, its purpose is to modify female breast, correction of ptosis, external expansion of the breast. If used the chest position on the design of ordinary women, will make the adjustment effect of lost bra bra pattern design; chest position should follow the anthropometric data adjusted, if the breast expanding bra pattern may be appropriate to chest ingression to gather effect; if the breast sagging phenomenon, can right chest point up the bra pattern so as to improve the effect of chest [9]. The distance between the breast height and the breast height is the two important parameters to evaluate the beauty of the female breast. The breast height determines whether the female breast tall and straight, the distance between the breast and the breast is on behalf of the female chest is outside the expansion and the extent of the external expansion.

\section{Human Body Measurement and Data Analysis}

Body measurement is the basic types of study and classification, in order to grasp the female breast shape characteristic, this research used 3D body scanning technology to obtain the data of human body, the combination of computer technology and the construction of digital clothing engineering increasingly close, three dimensional non-contact body scanning technology will become the future development trend of science and technology, an important step is the process of digital clothing customization production in.

Through the three-dimensional breast scanning image analysis of 223 young women in Shanghai, women get breast sizes, and using the scientific method of classification analysis, find out the wide coverage of representative female breast type. Accordingly, optimize the structure of the bra design, in order to find the best to adjust the breast shape to provide a basis for the structure.

\subsection{Human Body Measurement}

\subsubsection{Selection of Measurement Objects}

The human body measurements were randomly selected from 223 young and middle-aged women in Shanghai area, and the 3D human body was measured by ScanWox non-contact 3D laser scanner. In order to make the sample can reflect the overall characteristics of the sample has the following requirements: (1) randomly: each sample are randomly selected and not selected; (2) independence: each sample is independent existence, this effect does not affect other kind. The human body measurement of the extraction, as long as these two conditions, can be carried out random independent sampling [10].

\subsubsection{Measurement Requirements}

The measurement of indoor lighting and no closed; subjects 
naked hair with a net cover, not wearing any jewelry items; when measured by the professional staff under the guidance of the correct posture measurement test. The subjects of natural standing on the scanning platform, positioning mark at the feet on the scanning platform, arms drooping naturally, elbows slightly raised, elbow point slightly outwards. Hand relax, away from the thigh $8 \sim 10 \mathrm{~cm}$. When measuring, natural breathing, in front of the eye head, keep the body stable to avoid shaking [11].

\subsubsection{Measuring Position}

With reference to the relevant provisions of national standard «clothing body site of measurement and method», the final decision on the selection of women under the bust, milk spacing, chest width, higher milk as a measurement project.

In $3 \mathrm{D}$ body scanning map, you can get to a part of the data needed, such as height, chest circumference, some of the more detailed size need manual operation were measured in 3D scanning map. The measured position height, milk spacing, bust, bust, bust, on high, chest width, breast milk root point is high, the front neck point to point distance about milk.

\subsubsection{Measurement Results}

The purpose of this study is to find a representative of the strong, wide coverage of the chest type and system, scientific classification, according to the analysis of the results of the follow-up to facilitate the follow-up of women's bra version of the optimization design. The first is to find the largest number of cup type, narrow the scope of the study. Using the ScanWox non-contact three-dimensional laser human body scanner, 223 samples of random samples were processed by computer, and the data of more than and 100 human body related data can be obtained.

According to the classification of cup standard, the experimental samples are classified, AA cup A cup 10 people, 70 people, 75 people B cup, C cup D cup 44 people, 16 people, 8 people E cup. Thus the largest number of samples B cup. Then, the B cup subjects for detail size measurement, measurement items including milk point height, chest width, root height, breast milk spacing, chest circumference, chest, lower chest and neck point to the left and right front and back wide.

\subsection{Data Analysis}

In order to accurately analyze the data of human body 3D scanning, using SPSS data analysis method, first carries on the descriptive statistical analysis to determine the basic statistic clustering analysis; and then clustering analysis, classification of sample size and shape.

\subsubsection{Descriptive Analysis of Data}

Three basic statistics of sample mean value, standard value and variation coefficient were used to analyze the data of the pre processed data.

$$
\text { Sample mean: } \bar{X}=\frac{1}{n} \sum_{i=1}^{n} x_{i}
$$

According to the principle of mathematical statistics, the sample mean is the most commonly used statistic to describe the trend and the average level of the data set.

$$
\text { Standard deviation: } c . v=\frac{S}{\bar{X}} \times 100 \%
$$

Standard deviation is a characteristic value reflecting the degree of discrete random variables, the smaller the standard deviation, the lower the degree of sample dispersion, the more gathered in the vicinity of the expected value of the sample; on the contrary, the larger the standard deviation of the sample.

For the same part, you can use the standard deviation to compare the degree of dispersion of the data. But for different measurement sites, it is needed to calculate the variation coefficient to compare the degree of dispersion between different parts of the measured values.

Table 2. Mean, standard deviation and coefficient of variation of different parts.

\begin{tabular}{llll}
\hline & Mean & Standard deviation & Coefficient of variation \\
\hline Milk point high & 111.099 & 5.091 & 4.582399 \\
height & 156.993 & 5.4487 & 3.470664 \\
Chest width & 29.479 & 2.3193 & 7.867635 \\
Milk with a little high & 106.245 & 5.0633 & 4.765683 \\
Milk spot distance & 18.805 & 1.8581 & 9.880883 \\
Bust & 93.6377 & 7.70782 & 8.231535 \\
Front neck point to left chest & 29.2455 & 2.60377 & 8.903147 \\
Front neck point to right chest point & 28.8651 & 2.31728 & 8.027965 \\
Back width & 35.9056 & 3.214 & 8.95125 \\
\hline
\end{tabular}

As can be seen from the table, the data of each part of the overall dispersion is not large, can be determined by the mean, so you can choose SPSS in the mean clustering to classify the samples.

\subsubsection{Cluster Analysis}

We sort out the data into the SPSS software, the use of $\mathrm{K}$-means fast clustering classification method to classify the 75 samples, the target clustering is the three category, the results are as follows: table 3 : 
Table 3. Initial cluster center.

\begin{tabular}{llll}
\hline & Clustering & & \\
\cline { 2 - 4 } & $\mathbf{1}$ & $\mathbf{2}$ & $\mathbf{3}$ \\
\hline Milk point high & 111.5 & 102.6 & 119.8 \\
height & 153.6 & 147.4 & 165.2 \\
Chest width & 35.4 & 27.9 & 24.3 \\
Milk with a little high & 102.5 & 98.7 & 113.6 \\
Milk spot distance & 21.2 & 18.3 & 16.5 \\
Bust & 117.00 & 88.66 & 76.30 \\
Front neck point to left chest & 33.91 & 26.74 & 25.60 \\
Front neck point to right chest point & 33.41 & 28.49 & 24.92 \\
Back width & 41.60 & 35.33 & 30.82 \\
\hline
\end{tabular}

This table is the initial class center point specified by SPSS. It is quickly clustered into three classes and three classes are specified.

Table 4. ANOVA.

\begin{tabular}{lllllll}
\hline & Clustering & & Error & F & Sig. \\
\cline { 2 - 5 } & mean square & Df & mean square & df & & .000 \\
Milk point high & 558.983 & 2 & 11.111 & 72 & 50.308 & .000 \\
height & 503.541 & 2 & 16.525 & 72 & 30.471 & .795 \\
Chest width & 52.191 & 2 & 4.079 & 72 & 44.294 & .000 \\
Milk with a little high & 523.285 & 2 & 11.814 & 72 & 7.137 & .000 \\
Milk spot distance & 21.137 & 2 & 2.961 & 72 & 51.266 & .001 \\
Bust & 1291.360 & 2 & 25.190 & 72 & 20.928 & .000 \\
Front neck point to left chest & 92.216 & 2 & 4.406 & 72 & 19.813 & .000 \\
Front neck point to right chest point & 70.529 & 2 & 3.560 & 72 & 28.446 & .000 \\
Back width & 168.745 & 2 & 5.932 & & \\
\hline
\end{tabular}

F Tests should be used only for descriptive purposes, since the selected cluster will be used to maximize the difference between the different clusters in the case. The observed significance level does not change accordingly, so it can not be interpreted as a test of the hypothesis that the mean of the cluster is equal.

From the results of Table 4 analysis, we can see that the concomitant probability of the $\mathrm{F}$ statistic between the 3 classes is less than the significant level 0.01 . These three kinds of samples in milk high point, height, chest width, milk, milk with high point spacing, chest circumference, front neck point to the bust point, the left front neck point to right chest, back width of the nine significant difference, from the single factor analysis of variance of the 3 class. These 75 samples are divided into 3 types is successful. Each variable has a role to play, and it is effective for clustering.

Table 5. Final cluster center.

\begin{tabular}{llll}
\hline & \multicolumn{3}{l}{ Cluster analysis } \\
\cline { 2 - 4 } & $\mathbf{1}$ & $\mathbf{2}$ & $\mathbf{3}$ \\
\hline Milk point high & 111.7 & 105.5 & 114.5 \\
height & 158.2 & 151.6 & 160.0 \\
Chest width & 31.3 & 29.7 & 28.4 \\
Milk with a little high & 106.7 & 100.9 & 109.6 \\
Milk spot distance & 20.1 & 18.8 & 18.2 \\
Bust & 103.29 & 93.72 & 88.47 \\
Front neck point to left chest & 31.81 & 29.29 & 27.85 \\
Front neck point to right chest point & 30.89 & 29.29 & 27.51 \\
Back width & 39.46 & 35.78 & 34.11 \\
\hline
\end{tabular}

Table 5 is the final class center location, it can be seen that in the iterative process, the class center position with the transfer. At the same time, it can be seen that the SPSS software can be divided into three categories by the eleven iteration after the 75 samples. The table from left to right, first, because it is too short in height, chest circumference, can be called pyknic type; various parts of the data of second types of balanced symmetry, it can be divided into third types of symmetry; the highest height, bust minimum, this kind of sample called thin type [12].

Sample fast clustering method is used for large sample cluster analysis, can quickly disperse the various observation variables to the designated class. SPSS software will be 75 sample cluster into three categories, from left to right is chunky type, symmetrical type, thin type. The proportion of these three types of samples is shown in table 6 .

Table 6. The proportion of the three types of samples.

\begin{tabular}{lll}
\hline Body type & Sample quantity & Proportion \\
\hline Pyknic & 18 & $24 \%$ \\
Symmetrical type & 23 & $30.7 \%$ \\
Leptosome & 34 & $45.3 \%$ \\
\hline
\end{tabular}

\subsubsection{Chest Width Degree}

The bust and height ratio reflects the female chest circumference shape index, through the ratio of bust and height, the 75 samples can be divided into narrow chest type, standard type, type, type and appearance of chest [13]. 
Table 7. Chest type classification of tested samples.

\begin{tabular}{lllll}
\hline Chest circumference / height & $\leq \mathbf{0 . 4 9}$ & $\mathbf{0 . 5} \mathbf{0 . 5 3}$ & $\mathbf{0 . 5 3} \sim \mathbf{0 . 6}$ & $>\mathbf{0 . 6}$ \\
\hline Chest type & classification narrow chest & standard type & Beautiful type & broad chest type \\
\hline
\end{tabular}

Of the 75 samples tested, there were 2 people with a narrow chest type, accounting for $3 \%$ of the total sample, 3 of the standard type, $4 \%$ of the total sample, and 39 of the beautiful appearance, accounting for $52 \%$ of the total sample, and 31 of the broad chest type, accounting for $41 \%$ of the total sample. The largest number of the whole beautiful chest and broad chest, and basically the same. It can be found that young women in Shanghai area in the whole chest and chest slightly larger, expanding tendency.

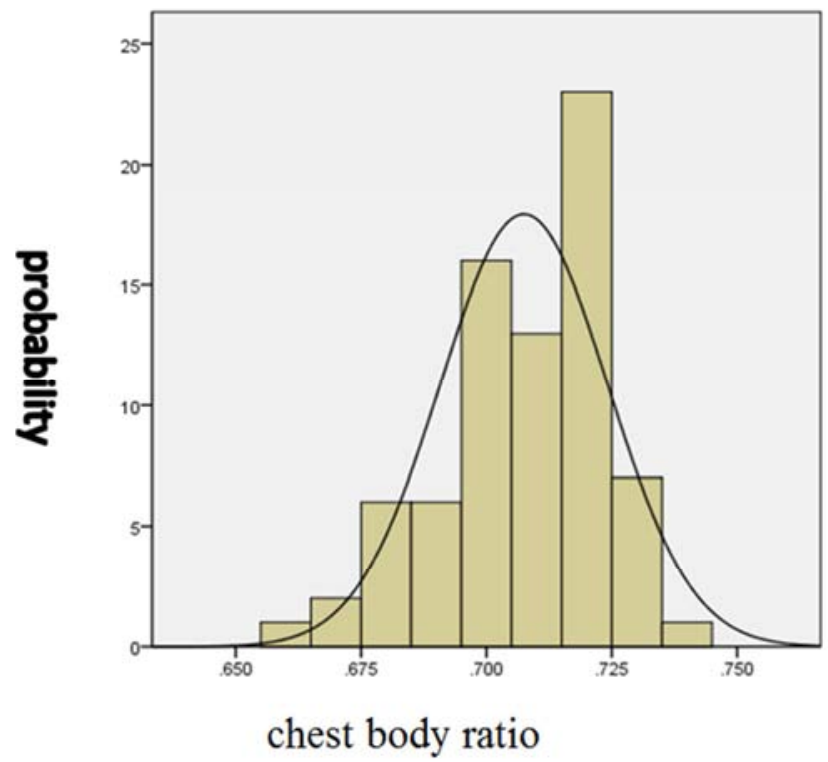

Fig. 1. Statistical frequency analysis of chest body ratio.

\subsubsection{Breast Height}

The ratio of chest height and height is the relative height of the female breast, the height of the breast point is the distance between the chest and the foot. If the female chest more tall and straight, the greater the chest than the contrary, if the smaller the ratio of the chest, then the breast closer to the waist line of women, the more serious sagging breasts. The ideal chest body ratio of common young women is 0.71 . In the SPSS data analysis, the data of the chest body ratio is described and the statistical frequency analysis is performed. Results as shown in Figure 1, the chest body ratio was 0.7 . The largest number of samples is $0.72 \sim 0.725$ this interval, followed by the number of $0.7 \sim 0.72$ range. From the results can be found in the Shanghai area of young women's breasts are relatively high degree of slight droop, but the degree of sag is not, a serious drop of the sample is not a lot.

\subsubsection{Breast Gather Degree}

Milk spacing and ratio of chest width to reflect the degree of the breast gather. As shown in Figure 2, the average value of the ratio of breast and chest width is 0.639 . In the tested samples, $80 \%$ of the subjects were concentrated in the $0.5 \sim 0.68$. If the milk and distance ratio of chest width is smaller, while breast gather degree is higher, whereas the external expansion is more serious. By 2 the histogram can be concluded that the existing external expansion to a certain extent breastshape whole young women in Shanghai area, but the expansion degree is not serious, can be corrected by adjusting the bra wearing the right, in order to maintain the appearance of breast. This requires the optimization of experimental bra design, better gather function. In bra pattern design optimization for the original chest inward push $0.5 \sim 1$ $\mathrm{cm}$, to gather effect.

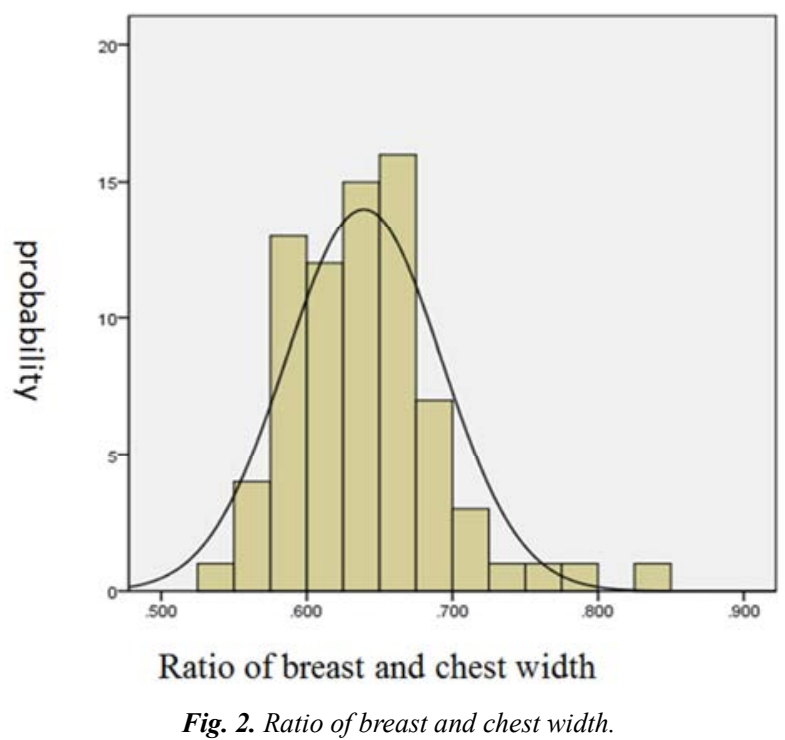

Previous studies found in the literature ideal distance is half milk chest width, and the ratio of breast in distance and milk before neck point to the bust point is just about the spacing of congruent triangles. Refer to the final cluster center table is not difficult to find that the 75 affected by the breast shape of different degrees of expansion and sagging, which is consistent with the previous data analysis.

\section{Conclusions}

The human body measurements were randomly selected from 223 young and middle-aged women in Shanghai area, and the 3D human body was measured by ScanWox non-contact 3D laser scanner. After computer processing, you can get more than and 100 of a human body according to the relevant data, this difference under the bust cup classification, according to the number of samples selected B cup of strong. The data analysis of 75 samples tested, the sample is divided into three categories, namely pyknic type, symmetrical type, thin type. According to the degree of chest width, the number can be found in the overall appearance type sample chest and chest type, and almost equal, young women in Shanghai area in the whole chest and chest slightly larger, expanding tendency; 
according to the analysis of relative breast height, can be found in young female breast in the Shanghai area, the relative height of slightly drooping, drooping degree not serious ptosis of the sample is also not many; according to the degree of breast can be found together, expanding to a certain extent breastshape whole young women in Shanghai area, but the expansion degree is not serious, can be corrected by adjusting the bra wearing the right, in order to maintain the appearance of breast.

\section{References}

[1] Duan xingyuan, Yu weidong. Study on the influence factors of bra wearing comfort [J]. Journal of Jiangsu Teachers University of Technology, 2007, 13 (4): 63-69.

[2] Liang suzhen, Zhang xin, Chen dongsheng. Body of the main detail size of bra structure design according to [J]. Textile journal, 2008, 29 (12): 69-73.

[3] Duan xiaojuan. Study on the adaptability of breast morphology and structure of the bra cup [D]. Beijing: Beijing Institute Of Fashion Technology, 2004.

[4] Zheng lingzi, Zhang daoying, Xi jinqi. Analysis of bra size based on the characteristics of female chest [J]. Guangzhou light industry, 2008 (5): 94-99.

[5] Liu xuan. Research on the structure of non steel ring bra [D]. Journal of Xi'an Polytechnic University, 2014.
[6] Chen huirong, Zhang xin, Tao na. Analysis of the characteristics of the breast shape of young women based on 3D human body measurement [J]. Journal of Xi'an Polytechnic University, 2008, 22 (2): 146-152.

[7] Liang suzhen. The basic shape of female college students in the south of China based on three dimensional human body measurement [J]. Journal of Xi'an Polytechnic University, 2009, 23 (4): 35-39.

[8] Wang jianping, zhangweiyuan, Gao shuping. A preliminary study on the thoracic morphology of young women [J]. Knitting industry, 2007 (7): 23-26.

[9] He yanjun, Xia xin, Zhou fengyuan. Three dimensional shape of the contour curve of the young women's milk [J]. Journal of Zhejiang Sci-Tech University, 2010, 27 (4): 561-565.

[10] Hyun-Young Lee, Kyunghi Hong. Optimal brassiere wire based on the 3D anthropometric measurements of under breast curve [J]. Applied Ergonomics, 2007 (38): 377-384.

[11] Liang suzhen, Zhang xin, Zhou jie. Basic shape of female college students in Western China based on three dimensional human body measurement [J]. Textile Journal, 2007, 28 (8): 75-78.

[12] Wang ping, Jin tiaofeng. The correlation analysis of the breast size of young and middle-aged women [J]. Journal of Zhejiang Textile and Fashion College. 2012 (04).

[13] Wang fangyuan, Luo yang, Wang jianping. Classification of thoracic morphology of young women in Shanghai [J]. Clothing, 2012 (4): 73-76. 